\title{
The Role of Ultrasound in the Assessment of Intrauterine Device Complications
}

\author{
Ahmed S Ansari, Thomas G Tullius J r, Jason R Ross, Sanja Kupesic Plavsic
}

\begin{abstract}
Over the pastfew decades, the use of intrauterine devices (IUDs) as long-term contraception has steadily been increasing. Along with the increased use of IUDs, many possible complications have been made apparent. This article presents an overview of normal IUD placement, possible complications and their diagnosis using ultrasound. Ultrasound has emerged as an excellent imaging modality for the diagnosis of many pelvic pathologies, because of its availability, sensitivity, specificity, cost-effectiveness and lack of radiation. By reading this article, the reader will be able to identify common IUD complications using ultrasound.
\end{abstract}

Keywords: Intrauterine device, Pelvic pain, Abnormal uterine bleeding, Contraception, Pelvic inflammatory disease, Uterine perforation, Uterine anomalies, Ultrasound.

How to cite this article: Ansari AS, Tullius TG J r, Ross J R, Plavsic SK. The Role of Ultrasound in the Assessment of Intrauterine Device Complications. Donald School J Ultrasound Obstet G ynecol 2012;6(3):318-326.

\section{Source of support Nil}

Conflict of interest: None declared

\section{INTRODUCTION}

Long-term intrauterine contraception (intrauterine contraceptive device, IUCD or intrauterine device, IUD) is currently the fastest growing and leading reversible contraception worldwide. ${ }^{1}$ In the United States there are two types of devices available, both are $T$ shaped, made of plastic, have a monofilament tail, and have barium sulfate in the frame to appear radiopaque. One form releases levonorgestrel and is functional for 5 years, and the other form is copper eluting (TCU380A) and is functional for 10 years. The other major class of modern IUD in mass use is the inert form that is either stainless steel, in use in the China market, or plastic loop with a core of barium sulfate, in use worldwide. Frameless variants of both eluting systems are available, but currently not in the United States. All forms of IUD in modern use can be assessed by ultrasound. Figures 1 to 4 show both transabdominal and transvaginal ultrasound images of correctly placed IUDs in various planes.

Careful counseling about contraceptive device complications, specifically side effects relating to bleeding disturbances, menstrual history, contraceptive history and general history and physical, have been shown to reduce the rates of postimplant dissatisfaction and complications. ${ }^{2}$

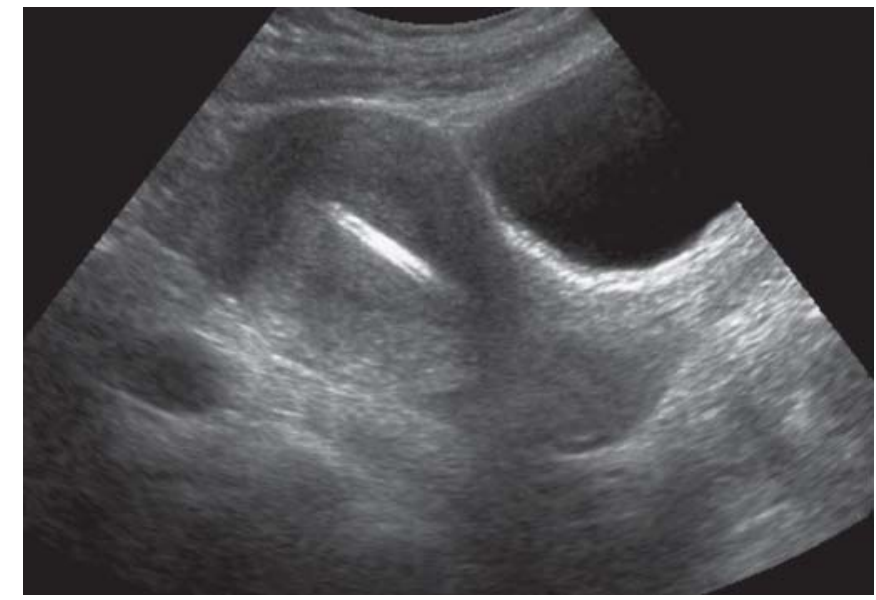

Fig. 1: Transabdominal ultrasound of IUD in place

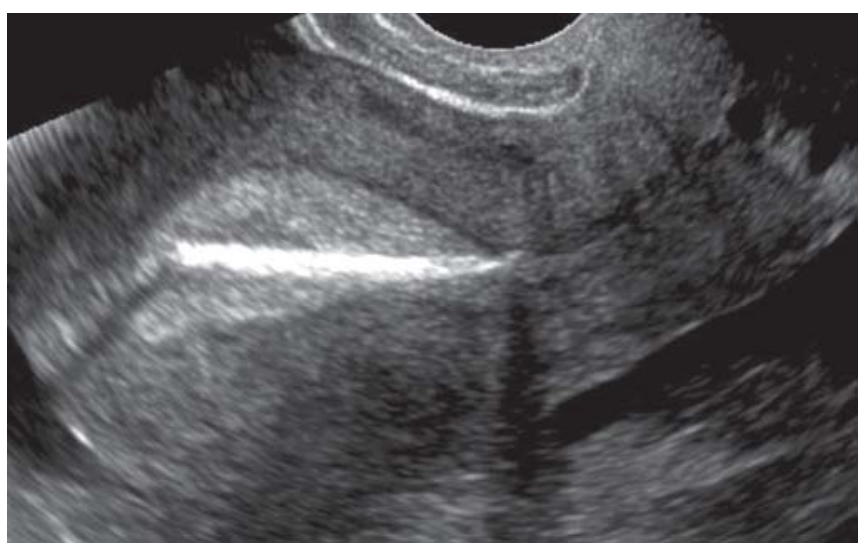

Fig. 2: Transvaginal ultrasound scan of an IUD in longitudinal plane

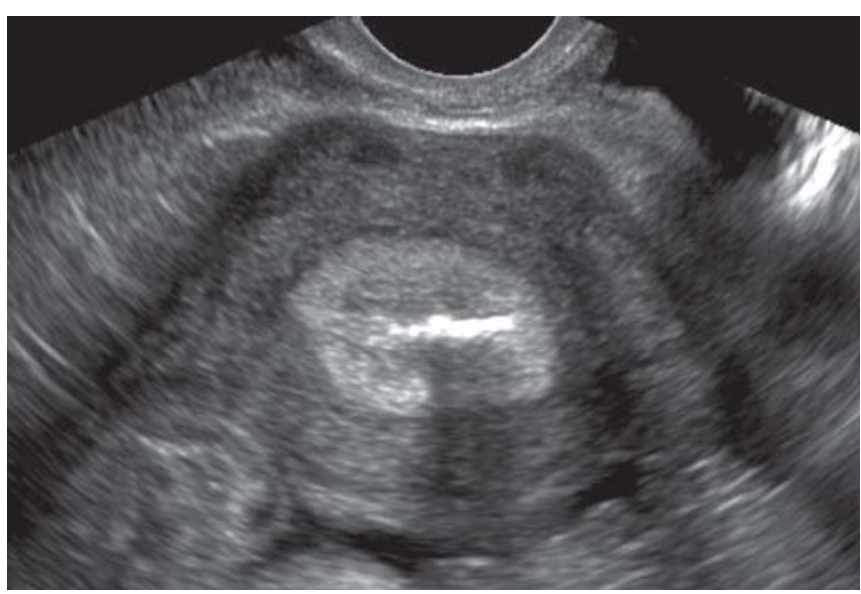

Fig. 3: Transvaginal ultrasound scan of an IUD in transverse plane

Currently the standard-of-care for normal insertion and use does not call for the use of an ultrasound. The first indication 


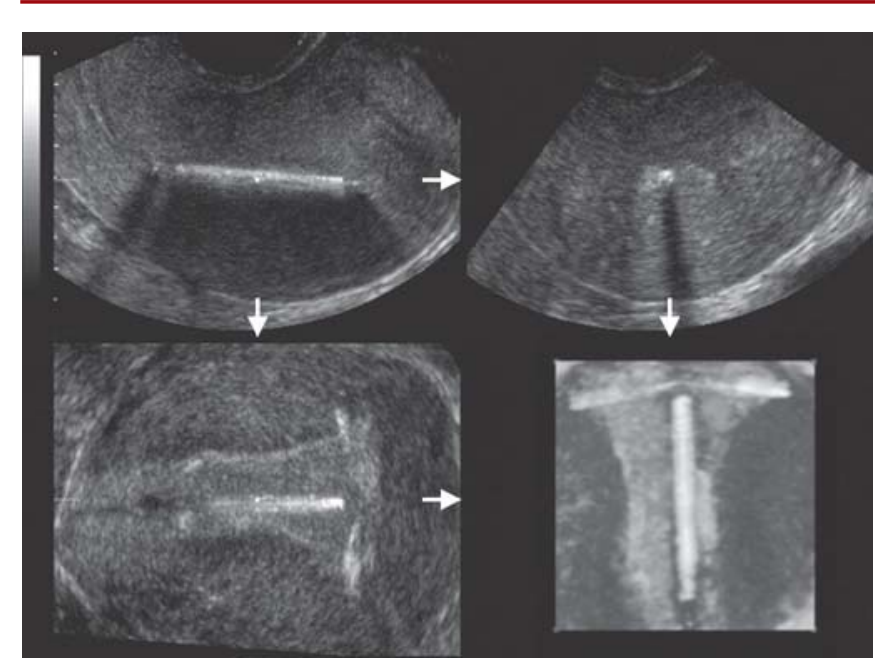

Fig. 4: Three-dimensional ultrasound and multiplanar imaging of an intrauterine device. Note correct placement of the T-shaped intrauterine device at the highest possible position within the uterine cavity

for use of ultrasound in IUD placement is the suspicion of uterine anomaly on speculum or uterine sound examination. If there is a suspected perforation, anomalies of the uterine fundus, or anomalies of uterine size then ultrasound analysis is indicated because any anatomical variant that interferes with proper placement is a contraindication to use, the rate of such congenital anomalies is roughly $3 \%$. The rate of acquired anomalies is hard to quantify because the size of anomaly that causes interference is up to clinician judgment. Some examples include:

- Massive uterine fibroids,

- Congenital uterine defects affecting the fundus, and

- Fundal fibroids interfering with placement.,4

A fter normal insertion, the majority of complications that require ultrasound analysis include symptomatic malposition, inability to find string after endocervical examination, and expulsion. The estimated rate of malposition approaches $10 \%$, but the majority of patients are asymptomatic and asymptomatic cases of malposition do not seem to increase the risk of pregnancy. When a malposition is found incidentally, the patient should be screened for signs and symptoms and then the decision about leaving it indwelling, replacing or terminating use are up to the clinician and patient preference. ${ }^{3}$ If the string cannot be found on endocervical examination or atypical symptoms of pain, cramping or bleeding develop then immediate pelvic ultrasound is the standard-of-care to evaluate the position or presence of the IUD. ${ }^{4}$ If the IUD is not found in the pelvis, the next step is to perform an upright $A / P$ and lateral $X$-ray to determine, if the IUD has migrated outside the uterus. If this has occurred, it is due to perforation. Perforation occurs in $0.1 \%$ of cases and is related to clinician experience, previous uterine trauma and uterine position and morphology. ${ }^{5}$ If the IUD is found in the myometrium the standard-of-care is generally operative hysteroscopy. If it is found outside the uterus, then laparoscopic retrieval becomes necessary. If the IUD cannot be found then intraoperative $\mathrm{X}$-ray or conversion to laparotomy becomes necessary. ${ }^{6,7}$

The management of an indwelling IUD during pregnancy is dependent on the trimester and IUD location. A fter the confirmation of an intrauterine pregnancy, one of the first steps is an ultrasound assessment to determine the location of the IUD and perform a fetal assessment. In either the first- or second-trimester, to retain the pregnancy, the risk of IUD removal must be weighed against the risk of an indwelling IUD. Currently, the data suggests that if the strings are visible on speculum examination then removal of the IUD in the first- or second-trimester posses less risk than leaving the IUD indwelling, as long as the removal is not likely to interrupt the placenta or membranes. ${ }^{7}$ If the strings are not visible in the first-trimester then ultrasound or hysteroscopic-guided removal is the safest option if it is feasible. ${ }^{6,7}$ In the early second-trimester, ultrasound analysis is needed to guide the clinical decision, if the strings are not visible. In the second-trimester, if the IUD is not in the gestational sac, in or behind the placenta, then removal is feasible. In the late second-trimester and third-trimester, if the strings are not visible, the IUD should be left in place. Other indications for ultrasound use in IU D therapy include difficult removal and replacement in cases of expulsion. Difficult to remove IU Ds can be appropriately managed with ultrasound guidance, often in the office. ${ }^{6}$ In cases of recurrent IUD expulsion, the patient should be evaluated for uterine anomalies. The reinsertion can be aided by ultrasound placement in difficult anatomy, and in cases, with multiple expulsions, the patient should be followed both clinically and by ultrasound every 6 months. ${ }^{7}$

\section{CASE REPORTS}

\section{Case 1}

A 27-year-old female (G3 P1203) presents to the emergency department with a 2-day history of cramping abdominal pain, nausea and vaginal spotting. She is currently sexually active with her husband and has an IUD in place for contraception. The IUD was placed 5 months ago. Onset of menarche was at age 11 with coitarche at 17 years. She has been treated successfully for a gonorrhea infection at age 17. Her last menstrual period was 3 weeks ago and was normal. Her pregnancy test is negative. Recently, she has complained to her husband of dyspareunia. Her past medical history includes childhood asthma and a tonsillectomy at age 7 . She has had 3 normal vaginal deliveries at age 19 , 


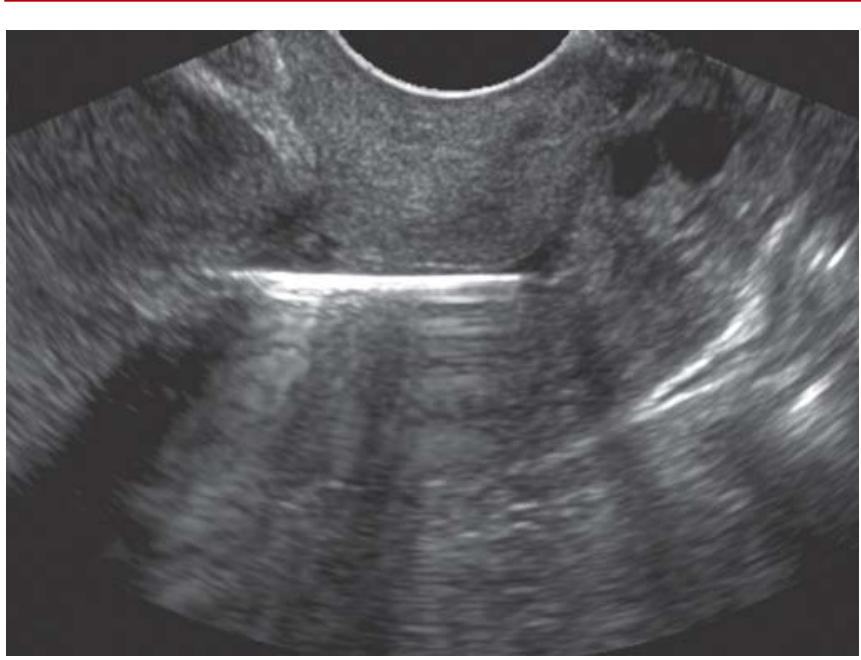

Fig. 5: Transvaginal ultrasound (transverse plane) of the uterocervical junction. Note low position of the IUD

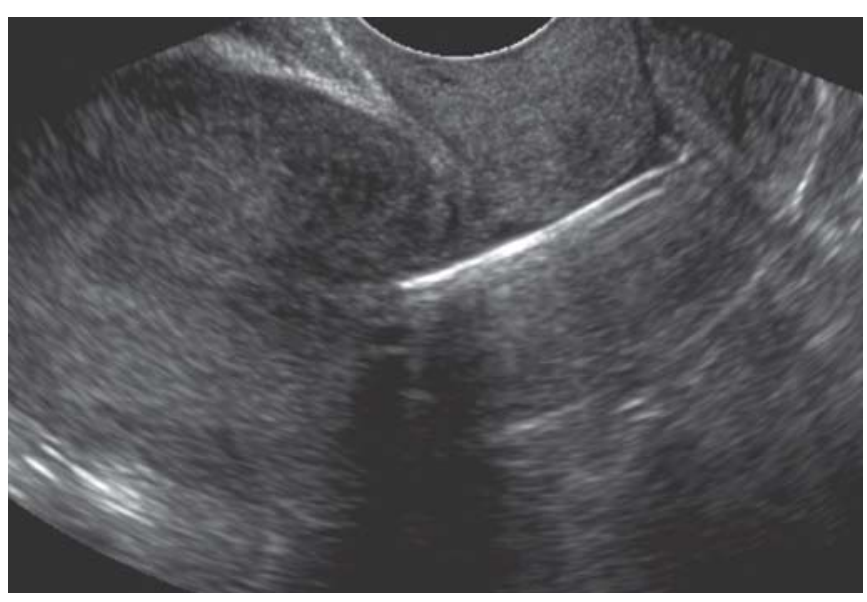

Fig. 6: Transvaginal scan of an IUD within the cervical canal (longitudinal plane)

23, 25 years, with the last two births at 34 and 35 weeks of gestation respectively. Her vital signs are: Blood pressure $120 / 85 \mathrm{~mm} \mathrm{Hg}$, pulse 90 beats per minute, respiratory rate 16 breaths per minute and temperature is $37^{\circ} \mathrm{C}\left(98.6^{\circ} \mathrm{F}\right)$. $\mathrm{Her}$ physical examination reveals mild bilateral lower quadrant tenderness and guarding. On pelvic examination, there is cervical motion tenderness, but no adnexal pain on either side. The remainder of her examination is normal. On speculum examination, a normal cervix is visualized and the strings of the IUD are easily seen. Figures 5 and 6 demonstrate her pelvic ultrasound findings.

\section{Case 2}

A 38-year-old female (G3P3) presents to your office with a one-day history of sharp right lower quadrant pain. The pain started this morning and has progressively worsened. She also complains of being nauseated and has vomited twice. Her history is significant for IUD insertion 1 week ago. Onset of menarche was at age 13, age of first intercourse

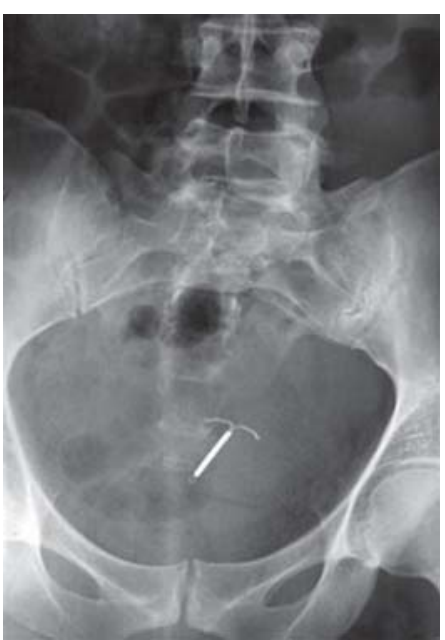

Fig. 7: Abdominal X-ray of a patient presenting with lower abdominal pain. Intrauterine device is visualized within the abdominal cavity. Laparoscopy revealed perforation of the uterus

was 22 years, with no history of sexually transmitted diseases. Her past medical history is unremarkable. Her vital signs are blood pressure $115 / 80 \mathrm{~mm} \mathrm{Hg}$, pulse 110 beats per minute, respiratory rate 20 breaths per minute and temperature is $37^{\circ} \mathrm{C}\left(98.6^{\circ} \mathrm{F}\right)$. Her physical examination is significant for bilateral lower quadrant tenderness, right adnexal enlargement and tenderness. The remainder of her examination is normal. Her urine pregnancy test is negative. Pelvic ultrasound reveals an empty uterus with no sign of the IUD placed 1 week ago. Figure 7 illustrates the abdominal $X$-ray of the patient.

\section{Case 3}

A 36-year-old female (G3P0121) presents to your office with vague lower left abdominal pain since the placement of an IUD 4 months ago in Juarez, M exico. The patient complains of mild cramping especially during her menses, but denies any abnormal vaginal bleeding or discharge. The patient is currently married and is sexually active with only her husband. Onset of menarche was at age 14, coitarche was at 18 years of age, and the patient denies any history of sexually transmitted diseases (STD). The patient's last menstrual period was 3 weeks ago and was normal. The patient's past medical history includes two spontaneous abortions at 14 and 15 weeks; due to financial inability, there was no workup to determine the causes of the abortions. The patient's other pregnancy was significant for preterm delivery at 34 weeks due to preterm labor. The patient's past medical history is unremarkable otherwise. The patient's vitals are blood pressure $107 / 68 \mathrm{~mm} \mathrm{Hg}$, pulse 71 beats per minute, respiratory rate 18 breaths per minute and temperature is $37.2^{\circ} \mathrm{C}\left(98.9^{\circ} \mathrm{F}\right)$. Her physical examination is unremarkable except for mild tenderness to palpation in the left lower abdomen. Figures 8 to 11 demonstrates her pelvic ultrasound findings. 


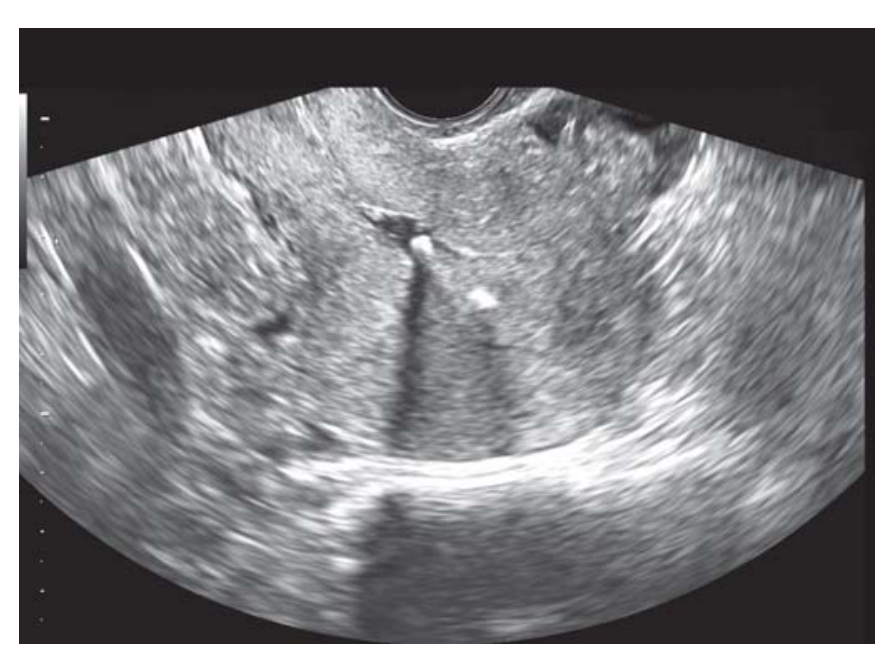

Fig. 8: Transvaginal ultrasound of the uterus with IUD. In longitudinal plane IUD is visualized within the uterine cavity. Small amount of intracavitary fluid is demonstrated in isthmic portion

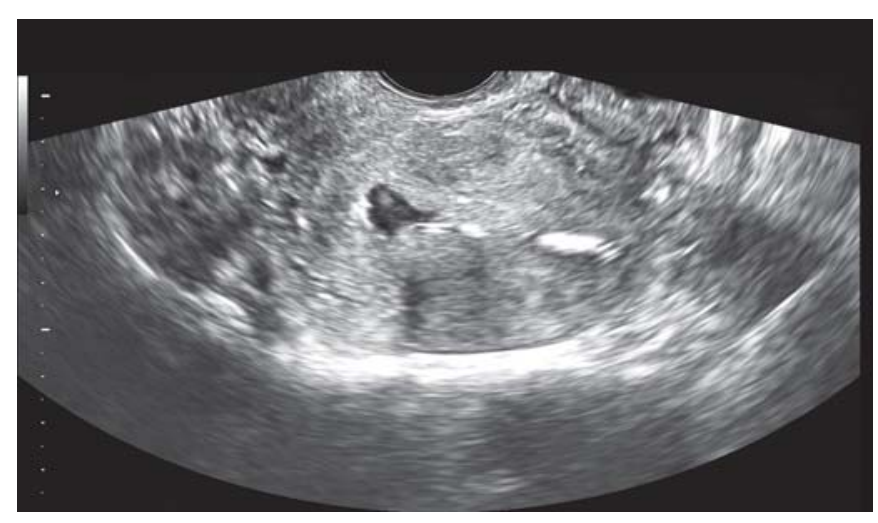

Fig. 9: Transverse plane illustrates both 'wings' of copper IUD. Small amount of intracavitary fluid is noted supracervically

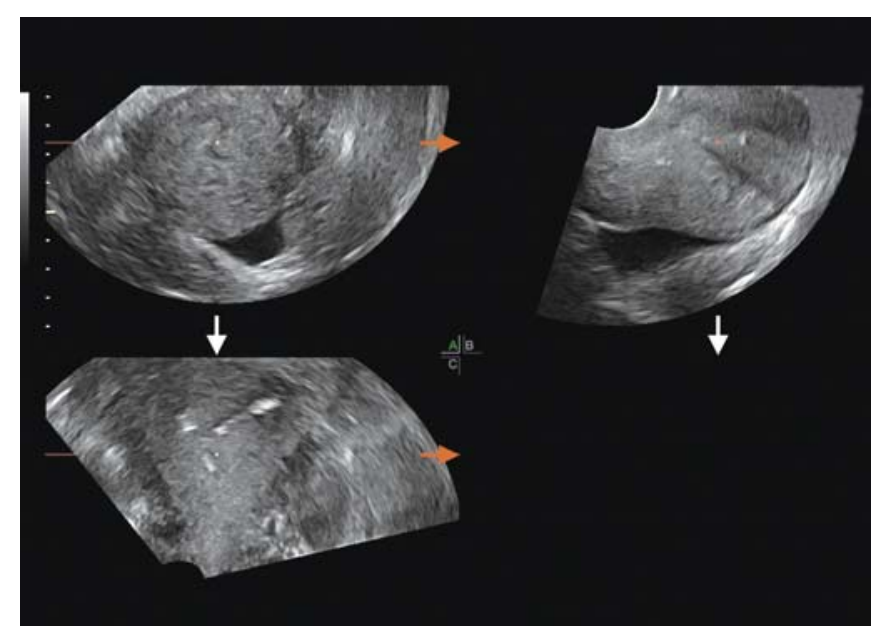

Fig. 10: Three-dimensional transvaginal ultrasound of the same patient revealed IUD malposition, clearly visualized in coronal plane (left lower image). The IUD was rotated upside down

\section{Case 4}

A 33-year-old female (G2P0020) presents to your office with dysuria, dyspareunia, vaginal discharge, postcoital spotting and pelvic pain for the past 1 month. The patient is

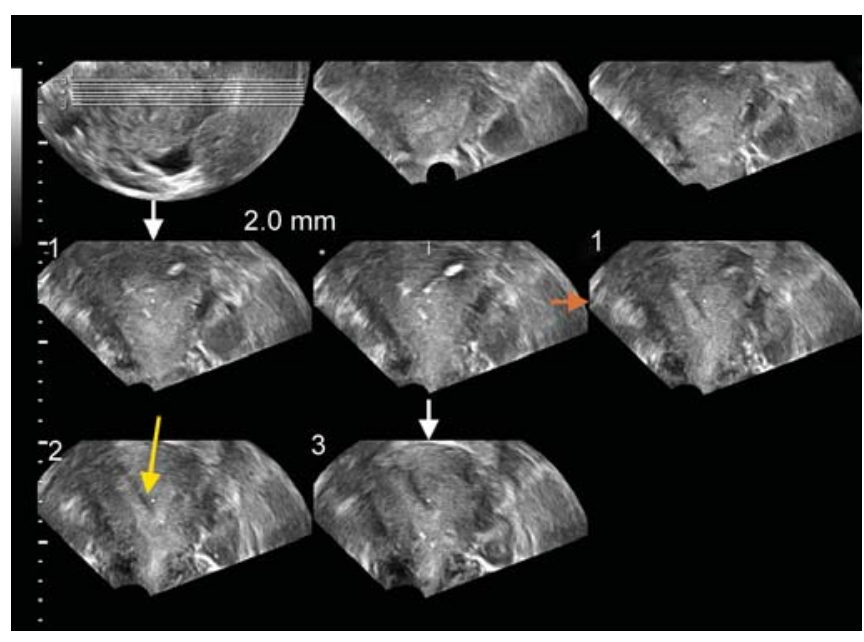

Fig. 11: Tomographic ultrasound imaging clearly demonstrated abnormal shape of the uterine cavity, typical for septate uterus (pointed by an arrow)

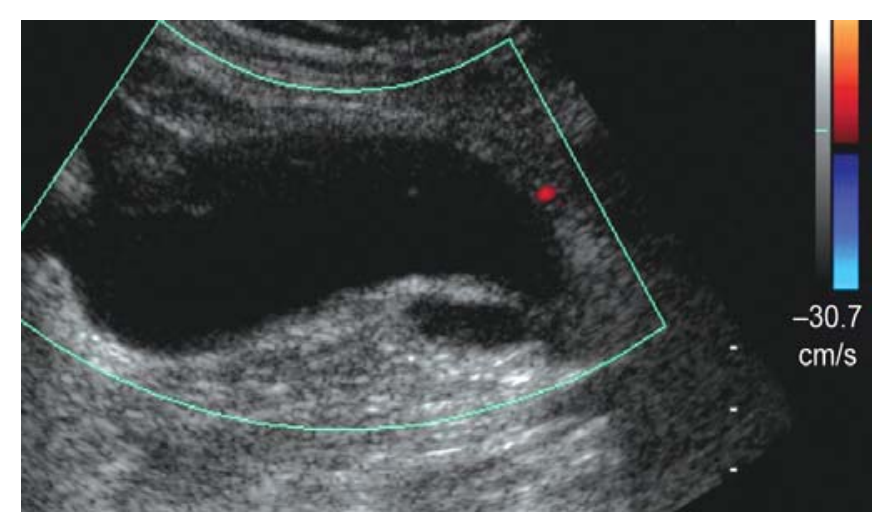

Fig. 12: Transvaginal sonogram of a complex adnexal mass containing a folded dilated tube and enlarged ovary in a patient with acute pelvic pain. The patient had IUD for 6 years. Medical history revealed a high-risk sexual behavior

currently single but is sexually active with several partners. Onset of menarche was at age 13 and coitarche was at 14 years of age. The patient states that she has been treated for several episodes of STD s in the past, but does not remember any details. The patient's last menstrual period was 3 weeks ago and was normal. The patient states that she had a copper IUD placed approximately 2 months ago and this is the only form of contraception that she uses. The patient's past medical history includes 2 elective abortions due to pregnancies being undesired. The patient's past medical history is unremarkable otherwise. The patient's vitals are blood pressure 120/70 mm Hg, pulse 98 beats per minute, respiratory rate 18 breaths per minute and temperature is $37.7^{\circ} \mathrm{C}\left(99.9^{\circ} \mathrm{F}\right)$. Her physical examination is remarkable for mild tenderness to palpation in the lower abdomen. On pelvic examination, copious purulent discharge was noted with a friable cervix and cervical motion tenderness. Transvaginal ultrasound detects an IUD in place. Careful exploration of adnexa reveals tubular sonolucent structures bilaterally (Fig. 12). 


\section{CASE DISCUSSION}

\section{Case 1: IUD in Cervix}

A 27-year-old female (G3 P1203) presents to the Emergency Department with a 2-day history of cramping abdominal pain, nausea and vaginal spotting. She is currently sexually active with her husband and has an IUD in place for contraception. B ased on the mild cramping, vaginal spotting, cervical motion tenderness, elongated IUD strings, dyspareunia and the ultrasound showing a malpositioned IUD, the patient most likely has an IUD located in the cervix.

Studies have shown that approximately 1 in 10 IUDs are mal positioned. ${ }^{8} \mathrm{~A} n$ IUD should be placed in the uterine fundus. Therefore, an IUD is considered malpositioned, if any portion is located in the lower uterine segment, myometrium, cervical canal or outside the uterus. To evaluate the location of the uterus, three-dimensional ultrasound has become a reliable tool. Copper IUDs are easily visualized with ultrasound while only a small portion of the levonorgestrel-secreting IUDs are detected by echo.

Our patient is symptomatic and the ultrasound showed a malpositioned IUD in the cervix. Therefore, the IUD should be removed. Because the IUD is located in the cervical canal, it should be removed as it may be expelled and undetected by the patient, leaving the patient at risk of becoming pregnant. To avoid a disruption in contraception, a new IUD may be placed at the same visit. The new IUD may be inserted under ultrasound guidance to ensure that a proper position is attained.

\section{Case 2: Uterine Perforation}

A 22-year-old female (G3P3) presents to the emergency department with 12 hours of progressive abdominal pain. The pain along with the history of IUD insertion 1 week ago, the absence of the IUD strings on speculum examination and the abdominal $X$-ray point toward uterine perforation by the IUD. IUD perforation occurs in about 1 in 1,000 IUD insertions and its risk is increased by practitioners inexperienced in IUD placement, retroverted uterus and uterus with anatomy defects. ${ }^{9}$ Patients often present with severe or chronic abdominal pain, vaginal bleeding and IUD strings that are greatly shortened or not visible. However, a perforation is not always obvious and clinicians often schedule a follow-up 4 to 6 weeks after insertion to rule out perforation. If the clinician suspects perforation, the patient should be monitored for signs of an acute abdomen. Watch for tachycardia or hypotension. Prophylactic antibiotics should be administered. If the exposed strings are present, gentle traction can be applied to them to attempt to localize the IUD. U Itrasound can be used to determine the position of the IUD as well and three- dimensional ultrasound is an excellent means of identifying a malpositioned IUD. ${ }^{10}$ If the IUD cannot be visualized, the IUD was most likely either expelled or perforated the uterus. A $n$ abdominal $X$-ray should be performed to rule out perforation. ${ }^{11}$ If perforation is detected, the next best course of action is to surgically remove the IUD to prevent perforation into abdominal viscera, bladder or blood supply. ${ }^{12}$ The first-line treatment for an IUD perforated into the abdomen is laparoscopy with laparotomy indicated only, if necessary. ${ }^{13}$

\section{Case 3: IUD Placement in Patient with Septate Uterus}

The patient in this case is a 36-year-old female (G 3P0121) with a history of recurrent miscarriages and preterm labor that started to have vague pelvic pain after the placement of an IUD. Her physical examination is insignificant. Unknown at the time of placement of the IUD, the patient had a septate uterus, which is a contraindication to the placement of an IUD.

U terine fusion defects are caused by failure of the right and left Müllerian ducts to fuse during embryogenesis. Incidence of uterine fusion defects is about $1 \%$ overall and $3 \%$ in females with poor reproductive outcomes. ${ }^{15}$ Depending on the extent of nonfusion, the result can lead to bicornuate uterus at the less extreme spectrum to a double uterus/cervix and even double vagina at the extreme spectrum, known as didel phys uterus. The etiology of septate uterus is somewhat different, because it develops secondary to incomplete resorption of the midline septum.

M ost patients with bicornuate uterus or septate uterus are usually asymptomatic. Patients are often diagnosed when they experience infertility, recurrent pregnancy loss or preterm labor/cervical incompetency. A mong uterine congenital anomalies, septate uterus is associated with the highest rates of infertility and spontaneous abortion rates. ${ }^{14}$ Rates of preterm delivery and spontaneous abortion can be as high as 19 and $42 \%$ respectively. ${ }^{14}$

U terine fusion defects are diagnosed by a combination of magnetic resonance imaging ( $\mathrm{MI}$ ), hysterosal pingogram and hysteroscopy. ${ }^{14}$ Transvaginal ultrasound can also be used for the diagnosis of uterine defects, but only threedimensional (not two-dimensional), is able to detect the abnormalities in question.

Currently the standard-of-care for normal insertion of an IUD does not call for the use of an ultrasound. The first indication for use of ultrasound in an IUD placement is the suspicion of uterine anomaly on speculum or uterine examination. A ny uterine anomal ies, such as uterine fusion defects, that interfere with the proper placement of an IUD is a contraindication to the use of an IUD. ${ }^{16,17}$ 
Figures 8 to 11 describe the patient described in case three. The first two images are two-dimensional transvaginal ultrasound images that show that this imaging modality is not very sensitive in the detection of uterine fusion defects. The last two images are three-dimensional and tomographic ultrasound images respectively, that show these imaging modalities are able to detect uterine cavity defects with a greater sensitivity.

\section{Case 4: IUD use in a Patient with High-Risk Sexual Behavior}

The patient in this case is a 33-year-old female (G2P0020) with a history of recurrent STD s that presents with dysuria, dyspareunia, vaginal discharge, postcoital spotting and pelvic pain for the past 1 month. Her symptoms are highly suspicious for pelvic inflammatory disease given her history of recurrent STDs. Her physical shows lower abdominal pain, copious cervical discharge with cervical friability and cervical motion tenderness. Figure 12 shows a transvaginal ultrasound verifying the suspected diagnosis of pelvic inflammatory disease.

Pelvic inflammatory disease (PID) is an inflammatory condition of the upper female reproductive system, including the uterus, fallopian tubes and ovaries. Classically, PID is caused by Neisseria gonorrhoae and Chlamydia trachomatis, but can also be caused by other unusual pathogens, such as E. coli. R isk factors for PID include early coitarche, multiple sexual partners, nonbarrier contraception and history of past STDs. Classically, PID presents with fever, pelvic pain and adnexal tenderness. On pelvic examination classic signs include cervical motion tenderness, purulent discharge and friability. The gold standard for the diagnosis of PID is laparoscopy, but is rarely used as often the physical examination and history are sufficient to make the diagnosis. The use of a transvaginal ultrasound can also help support the diagnosis, as in this patient. Complications of PID include infertility due to pelvic, ovarian and tubal adhesions; tubo-ovarian abscess; Fitz-Hugh-Curtis syndrome, and chronic pelvic pain.

In the past it was believed that the use of an IUD significantly increased the risk of PID. This was true in the past, as a braided string was used in IUDs; however, currently monofilament strings are used. The braided string allowed bacteria a means of ascending the female genital tract to go on and infect the uterus, fallopian tubes and ovaries. Current studies indicate that patients are at only a slightly increased risk of PID above the general public for the 3 months after the placement of an IUD. ${ }^{20}$ The current overall incidence of PID in patients with an IUD in place is 6 per $1,000 .{ }^{20}$ The risk of PID correlates with the prevalence of STDs in the given population. ${ }^{19}$ Incidence of PID in patient with an IUD is especially higher in populations with a higher prevalence of STDs and lack of adequate access for STD screenings. ${ }^{19}$ Current contraindications to IUD placement are current PID, current purulent cervicitis or chlamydial or gonococcal infection. ${ }^{21}$ In patients that have signs and symptoms of PID or cervicitis, appropriate diagnostic testing should be done to rule out possible PID or cervicitis. ${ }^{21}$ At one time, antibiotic prophylaxis was advocated, but current studies show that antibiotic prophylaxis is inconclusive. ${ }^{18,19}$ Currently, there are no recommendations on routine ST D screening in patients with IUDs, how ever, close follow-up is advised. ${ }^{18,21}$ A cute PID is not an indication for the removal of an IUD. ${ }^{18}$

\section{SE LF-ASSESSMENT QUIZ}

1. An 18-year-old G OPO C aucasian female comes into your office wanting a reliable long-term contraception. She wants something she does not have to think about every day. She is in college and plans on attending medical school. She would like a contraceptive device that will be effective until well into residency. She knows that she wants an IUD and has heard that she needs to be 'tested' before she can receive one. What is an appropriate radiographic preimplantation study?
A. Ultrasound
B. X-ray pelvic
C. X-ray $A / P$ and lateral
D. Ultrasound
E. None

2. A 32-year-old G2P2 Hispanic female comes to your office with generalized pelvic pain. She had lower right quadrant pelvic pain for a few weeks that has progressed to the current generalized symptoms. Y ou perform a pelvic ultrasound and do not see any pelvic pathology. In fact, you do not see anything in the pelvic cavity and this is very concerning. She is one of your regular patients, and you know that she has an IUD. What is the next step in the management of this patient?
A. Ultrasound
B. CT
C. Pelvic X-ray
D. $A / P$ and lateral $X$-ray
E. Laparotomy 
3. A 24-year-old G1P1 Caucasian female comes to the hospital complaining of morning nausea and vomiting. Her beta-hCG is found to be 4,500 . She tearfully tells you that she 'cannot be pregnant because she has an IUD that was only implanted 4 months ago.' On speculum examination, you observe a bluish friable cervix with a monofilament string protruding approximately $2 \mathrm{~cm}$. On ultrasound the IU D presents in the lower third of the uterus. What is the appropriate management?
A. Remove the IUD
B. Explain that the procedure to remove the IUD has
more risk associated with it than leaving it in place
C. Removal in the OR with US guidance
D. Clip the monofilament off at the level of the cervix

4. A 24-year-old GIP1 Caucasian female comes to the hospital complaining of morning nausea and vomiting. Fetal heart tones are heard and the fundal height is found at the umbilicus. She tearfully tells you that she cannot be pregnant because she has an IUD that was only implanted 14 months ago. On speculum examination you observe a bluish friable cervix. What location would preclude a removal of the IUD?
A. L ower third of the uterus
B. Behind the placenta
C. Fundal region
D. Vaginal wall

5. A nulliparous 21-year-old female presents to the emergency department with a 12 hours history of cramping abdominal pain, nausea and vaginal spotting. She is currently sexually active with 3 male partners and has an IUD in place for contraception. U rine betahCG is negative. Her vital signs are blood pressure 111/ $74 \mathrm{~mm} \mathrm{Hg}$, pulse 83 beats per minute, respiratory rate 20 breaths per minute and temperature is $37^{\circ} \mathrm{C}\left(98.6^{\circ} \mathrm{F}\right)$. Her physical examination reveals mild left lower quadrant tenderness and muscle contractions. On pelvic examination, there is no adnexal pain or masses noted on either side. On speculum examination, a normal cervix is visualized and elongated strings of the IUD are found. W hat is the next diagnostic step to determine the cause of the abdominal pain and spotting?
A. A bdominal X-ray series
B. Complete blood count
C. A bdominal and pelvic CT scan with contrast
D. Pelvic ultrasound
E. Urinalysis

6. A 24-year-old female (G 4 P4) presents to the emergency department with 24 hours of progressive abdominal pain. The pain is currently in the left lower quadrant, and has been associated with nausea and one episode of vomiting. She reports no vaginal bleeding or discharge. She is currently sexually active with her husband and is using a copper IUD for contraception. Her urine betahCG is negative. $V$ itals signs are blood pressure 115/72 $\mathrm{mm} \mathrm{Hg}$, pulse 100 beats per minute, respiratory rate 22 breaths per minute and temperature is $37^{\circ} \mathrm{C}\left(98.6^{\circ} \mathrm{F}\right)$. Physical examination findings include bilateral lower quadrant pain. Her pelvic examination is normal. On speculum examination, no strings are visualized. Pelvic ultrasound fails to locate the IUD. What testing should be conducted next?
A. A bdominal $X$-ray series
B. Complete blood count
C. A bdominal and pelvic CT scan with contrast
D. Repeat pelvic ultrasound in 12 hours
E. Urinalysis

7. A 33-year-old female (G1 P1) presents to the Emergency Department with 12 hours of progressive abdominal pain. The pain is currently in the left lower quadrant, and has been associated with nausea and one episode of vomiting. She reports no vaginal bleeding or discharge. She is currently sexual ly active with two male partners and is using a copper IUD for contraception. Her urine beta-hCG is negative. She reports no dysuria. Vitals signs are blood pressure $95 / 64 \mathrm{~mm} \mathrm{Hg}$, pulse 87 beats per minute, respiratory rate 20 breaths per minute and temperature is $37^{\circ} \mathrm{C}\left(98.6^{\circ} \mathrm{F}\right)$. Her pelvic examination finds no cervical motion tenderness or adnexal masses. On speculum examination, there is a normal appearing cervix without discharge. No strings are visualized. The remainder of her physical examination is within normal limits. Pelvic ultrasound is unable to visualize the IUD. Her abdominal $X$-ray findings are an IUD located in her abdominal cavity. Which of the following is not a risk factor leading to this condition?
A. Inexperienced practitioner
B. Retroverted uterus
C. Patient with a history of a treated Chlamydia infection
D. Uterus with an anatomic defect
E. Cervical stenosis

8. A 26-year-old female GOP0000 patient presents to your office desiring contraception, specifically an IUD. The patient is in a long-term monogamous relationship with her boyfriend of 6 years. The patient's last menstrual period was 2 weeks ago and was normal. The patient's past medical history is significant for PID 8 years ago. The patient's family history is significant for bicornuate 
The Role of Ultrasound in the Assessment of Intrauterine Device Complications

uterus in her mother. The patient is currently asymptomatic and her physical and pelvic examinations are unremarkable. What is the next best course of action?

A. A gree to place an IUD device after appropriate counseling on risks and benefits

B. Order CT imaging to rule out bicornuate uterus

C. Tell the patient that IUD placement is contraindicated for her as she is too young

D. Tell the patient that IUD placement is contraindicated for her as she has a past medical history of PID

9. A 30-year-old female GOP0000 patient well known to you presents to your office desiring contraception, specifically an IUD. The patient recently married her boyfriend of 3 years and is only sexually active with her husband. The patient's last menstrual period was 3 weeks ago and was normal. The patient's past medical history is unremarkable except for three episodes of bacterial vaginosis in the past 1 year. The patient is currently asymptomatic. On physical examination the patient has adnexal and suprapubic tenderness. On pelvic examination she has a friable cervix and cervical motion tenderness. What is the next best course of action?

A. A gree to place an IUD device after appropriate counseling on risks and benefits

B. Order appropriate tests to rule out current PID or cervicitis

C. Tell the patient that IUD placement is contraindicated for her as she is nulliparous

D. A gree to place an IUD device but inform the patient that she is at increased risk for pelvic inflammatory disease since, she has had multiple episodes of bacterial vaginosis

10. A 27-year-old female G3P3003 presents to your clinic desiring long-term reversible contraception. A this time the patient only complains of moderate pain and bleeding during her menses. The patient's past medical history is unremarkable and physical examination is unremarkable as well. On pelvic examination, you see some yellowishgreenish discharge from the cervix. The patient is currently single and has recently begun a new sexual relationship and is only sexually active with this new partner. After discussion of various types of contraception, she decides that she would like the IUD. What is the appropriate response to this decision?

A. Agree to place an IUD device after appropriate counseling on risks and benefits.

B. Advise the patient that she would benefit greatest from the progesterone secreting IUD, but only after ordering appropriate tests to rule out current PID or cervicitis.

C. Tell the patient that IUD placement is contraindicated for her as she may have cervicitis or PID and even after treatment the placement of the IUD is still contraindicated.

D. Give the patient prophylactic antibiotics for possible cervicitis and proceed to place the IUD.

\section{Correct Answers}

1: $E ; 2: D ; 3: A ; 4: B ; 5: D ; 6: A ; 7: C ; 8: A ; 9: B ; 10: B$

\section{REFERENCES}

1. Population Reference Bureau. Family Planning Worldwide, 2002 Data Sheet. http://www.prb.org/pdf/F amPlanW orlwide_ Eng.pdf

2. Darney PD, K laisie CM . Contraception-associated menstrual problems: Etiology and management. Dialogues Contracept 1998;5:1.

3. Braaten $K P, B$ enson $C B, M$ aurer $R$, Goldberg $A B$. $M$ al positioned intrauterine contraceptive devices: Risk factors, outcomes, and future pregnancies. Obstet Gynecol 2011;118(5):1014.

4. Peri N, Graham D, Levine D. Imaging of intrauterine contraceptive devices. J Ultrasound M ed 2007;26(10):1389.

5. M echanism of action, safety and efficacy of intrauterine devices. Report of a WHO Scientific Group. World Health Organ Tech Rep Ser 1987;753:1.

6. Turok DK, Gurtcheff SE, Gibson K, Handley E, Simonsen S, $M$ urphy PA. Operative management of intrauterine device complications: A case series report. Contraception 2010;82(4):354.

7. Thonneau $P, A$ Imont $T$, de $L$ a R ochebrochard E, M aria B. Risk factors for IUD failure: Results of a large multicentre casecontrol study. Hum Reprod 2006;21(10):2612.

8. Braaten $K P, B$ enson $C B, M$ aurer $R$, Goldberg $A B$. $M$ al positioned intrauterine contraceptive devices: Risk factors, outcomes, and future pregnancies. Obstet Gynecol 2011;118(5):1014-20.

9. A ndersson K, Ryde-B lomqvistE, Lindell K, Odlind V, M ilsom. Perforations with intrauterine devices. Report from a Swedish survey. Contraception 1998;57(4):251.

10. Gill RS, M ok D, Hudson M, Shi X, Birch DW, Karmali S. L aparoscopic removal of an intra-abdominal intrauterine device: case and systematic review. Contraception 2012;85(1):15.

11. $M$ arkovitch $O, K$ lein $Z$, Gidoni $Y$, Holzinger $M$, Beyth $Y$. Extrauterine mislocated IUD: Is surgical removal mandatory? Contraception 2002;66(2):105.

12. B enacerraf BR, Shipp TD, Bromley B. Three-dimensional ultrasound detection of abnormally located intrauterine contraceptive devices which are a source of pelvic pain and abnormal bleeding. Ultrasound Obstet Gynecol 2009; 34(1): 110-15.

13. Peri N, Graham D, Levine D. Imaging of intrauterine contraceptive devices. J Ultrasound M ed 2007;26(10):1389-401.

14. Falcone: Clinical Reproductive M edicine and Surgery (1st ed). Online Version.

15. $M$ arcal L, N othaft M A, Coel ho F, V ol pato R, Iyer R. M ullerian duct anomalies: $M R$ imaging. A bdom Imaging 2011;36(6): 756-64.

16. Understanding and using the U.S. M edical Eligibility Criteria for Contraceptive Use, 2010 Committee Opinion.http:// ww w. acog.org/Resources_And_Publications/ Committee Opinions/Committee on_Gynecologic Practice/ Understānding_and_Using_the_US_M édical_ Eligibility_Criteria_for_Contraceptive_U sé_2010

17. Simón $C^{-}, M$ artinez $L^{-}$, Pardo $F$, Tortajad̄a $M$, Pellicer $A$. $M$ üllerian defects in women with normal reproductive outcome. Fertil Steril 1991;56(6):1192. 
18. Pelvic Inflammatory Disease. A m Fam Physician 2012 A pril;96(1):5-8.

19. Steen $R$, Shapiro K. Intrauterine contraceptive devices and risk of pelvic inflammatory disease: Standard of care in high STI prevalence settings. Reprod Health M atters 2004;12(23): 136-43.

20. Intrauterine devices: A $n$ effective alternative to oral hormonal contraception. Prescrire Int 2009;18(101):125-30.

21. Martínez F, López-A rregui E. Infection risk and intrauterine devices. A cta O bstet Gynecol Scand 2009;88(3):246-50.

\section{ABOUT THE AUTHORS}

\section{Ahmed S Ansari}

Student Physician, Department of M edical Education, Paul L Foster School of M edicine, Texas Tech U niversity, EI Paso, Texas, USA

\section{Thomas G Tullius J $r$}

Student Physician, Department of M edical Education, Paul L Foster School of Medicine, Texas Tech U niversity, EI Paso, Texas, USA

\section{J ason R Ross}

Student Physician, Department of M edical Education, Paul L Foster School of M edicine, Texas Tech U niversity, EI Paso, Texas, USA

\section{Sanja Kupesic Plavsic (Corresponding Author)}

Professor, D epartment of Obstetrics/Gynecology and Radiology, Paul L Foster School of M edicine, Texas Tech University, 4800 A lberta A ve, EI Paso, Texas 79905, USA, Phone: +1 (915)783-1700 e-mail: sanja.kupesic@ttuhsc.edu 